\title{
An Automated Carotid Pulse Assessment Approach Using Doppler Ultrasound
}

\author{
Alfred C. H. Yu*, Member, IEEE, Eric Cohen-Solal, Balasundar I. Raju, and Shervin Ayati
}

\begin{abstract}
During cardiac arrest emergencies, lay rescuers are required to manually check the patient's carotid pulse after the delivery of defibrillation shocks to assess the cardiac resuscitation progress of the patient. As a more automated way of monitoring the resuscitation progress, a new Doppler-ultrasound-based carotid pulse assessment approach is presented in this paper. The method works by analyzing the temporal aperiodicity of Doppler shifts seen in the ultrasound echoes returned from the patient's carotid arteries. As a quantitative investigation with this method, we derived a new measure called the pulselessness indicator to assess whether a carotid pulse is absent based on the given Doppler information. To study the performance of the new carotid pulse checking method, we built a multi-channel CW Doppler prototype device to acquire Doppler data in vivo during cardiac arrest experiments conducted on five different swines and computed pulselessness indicator estimates with these data. Our results indicated that the Doppler-based pulse checking approach has good sensitivity and specificity: it had a pulselessness detection rate greater than 0.9 for a given false alarm rate of 0.05 . As a further analysis, the prototype device was applied to other experiments where the swine had suffered cardiac arrest for over five minutes. It showed a consistent assessment performance on the monitoring of the swine's resuscitation progress after defibrillation and chest compressions.
\end{abstract}

Index Terms-Cardiac arrest, cardiopulmonary resuscitation, carotid pulse check, Doppler ultrasound, pulselessness.

\section{INTRODUCTION}

$\mathbf{S}_{\mathrm{a}}^{\mathrm{u}}$ UDDEN cardiac arrest is a health disorder related to the abrupt dysfunctioning of the heart. This health problem is well-recognized as one of the leading causes of death in the United States and other developed countries [1]. In terms of the problem triggers, it is traditionally considered that many of the cardiac arrests are attributed to ventricular fibrillation, but clinical statistics have recently revealed that an increasing number of occurrences are linked to the development of pulseless electrical activity (PEA) [2], [3]. While ventricular fibrillation and ventricular tachycardia are treatable through defibrillation therapy, such a treatment is not suitable for cardiac arrest patients who have developed PEA due to the dissociation between the electrical and mechanical activities of the heart.

Manuscript received March 26, 2007. Asterisk indicates corresponding author.

*A. C. H. Yu is with the Department of Electrical and Electronic Engineering, The University of Hong Kong, Chow Yei Ching Building Rm. 601, Pokfulam Road, Hong Kong SAR (e-mail: alfred.yu@eee.hku.hk)

E. Cohen-Solal and B. I. Raju are with Philips Research North America, Briarcliff Manor, NY 10510 USA

S. Ayati is with Philips Medical Systems, Andover, MA 01810 USA.

Color versions of one or more of the figures in this paper are available online at http://ieeexplore.iee.org.

Digital Object Identifier 10.1109/TBME.2007.908104
In these PEA cases, lay rescuers can only treat the patient by administering cardiopulmonary resuscitation (CPR) in order to "buy time" while waiting for the ACLS (clinical acronym for "Advanced Cardiac Life Support") team to intervene.

To assess whether blood circulation has been resumed in the patient after a cardiac arrest, lay rescuers are required to manually check the patient's carotid pulse following a CPR procedure or a series of defibrillation shocks [4]. From the diagnostic outcome of the carotid pulse check, they can then decide if further CPR is needed. However, many studies have suggested that the manual pulse check is an unreliable diagnostic test because lay rescuers usually fail to identify pulselessness (or the lack of circulatory pulse) within the recommended diagnostic time of $10 \mathrm{~s}$. For instance, Eberle et al. [5] have pointed out that only $2 \%$ of the lay rescuers in their study were able to identify pulselessness correctly within $10 \mathrm{~s}$. Moule [6] has also shown similar results where only 9 of the 49 lay rescuers accurately detected pulselessness within the $10 \mathrm{~s}$ time interval. More recently, Lapostolle et al. [7] have indicated that even for paramedics, the likelihood of correct pulselessness detection using the manual pulse check was merely $58 \%$. Since the failure to identify pulselessness would significantly reduce the patient's chances of survival (due to the lack of further CPR), it is important to devise a reliable resuscitation monitoring approach that gives more consistent performance than the manual pulse check.

Some studies have considered the development of automated methods for resuscitation monitoring on a cardiac arrest patient [8]. Perhaps the most common of these methods is to perform waveform analysis on the electrocardiogram (ECG) data that is available from the automated external defibrillator. As pointed out by Hallaway and Menegazzi [9], many investigations have studied the changes in the ECG waveform structure before and after defibrillation shocks as well as CPR procedures in order to predict the success of the treatment. However, as suggested by Niemann et al. [10], ECG data analysis may not be a completely reliable resuscitation monitoring approach by itself because it is inherently not capable of recognizing PEA development in the patient. Another way to monitor resuscitation is to use capnography to measure the patient's end-tidal carbon dioxide $\left(\right.$ ET- $\left.\mathrm{CO}_{2}\right)$ level. This method, as reviewed by Morimoto et al. [11], is generally a good indicator for the patient's return of spontaneous circulation, but it is not always effective in detecting the absence of circulation (i.e., it cannot reliably detect pulselessness) because the ET- $\mathrm{CO}_{2}$ level may significantly differ between cardiac arrest patients. A third possible monitoring approach, which is recently proposed by Pellis et al. [12], is to measure the transthoracic electrical impedance that varies during cardiac contraction and breathing. Nevertheless, 
this method can only provide an indirect indication for the return of circulation, and it has so far been studied merely in the context of cardiac arrest detection.

In this paper, we present the development of a new Doppler-based carotid pulse assessment approach as an alternative way to automatically and non-invasively monitor resuscitation progress. Our method, which is based on an initial framework that we recently reported [13], works by analyzing the time-varying Doppler spectral contents seen in the ultrasound echoes returned from the carotid arteries. As a quantitative investigation with our method, we derived a new normalized measure called the pulselessness indicator to study the level of pulselessness from the Doppler information. This new measure is based on the degree of aperiodicity seen in the Doppler spectral contents.

The rest of this paper is organized as follows. First, we provide an overview of the Doppler experimental setup that we developed for carotid pulse assessment. After that, we present the derivation of the new pulselessness indicator and discuss how this measure can be used to study the level of pulselessness in a patient's carotid arteries. Subsequently, we describe some cardiac arrest experiments that were performed in an in vivo setting and present the pulselessness detection results that we obtained with our Doppler-based pulse checking approach.

\section{Carotid Pulse Assessment Using DOPPLER ULTRASOUND}

\section{A. Fundamental Principles}

It is well established that Doppler ultrasound can be used to measure flow velocities in the vasculature. This flow measurement approach works by measuring the Doppler frequency shifts between the transmitted ultrasound waves and the echoes returned from moving scatterers. From the Doppler equation $[14$, Ch. 3], it is known that the frequency shift magnitudes are dependent on the scatterer velocity distribution and the cosine of the beam-flow angle. Based on this relationship and the fact that a periodic velocity distribution is only seen during regular cardiac activity, we can detect pulselessness by studying the level of temporal aperiodicity observed in the Doppler frequency contents (as long as the beam is not perpendicular to the flow direction). This principle essentially forms the working basis of our Doppler-based carotid pulse assessment approach.

\section{B. Design Considerations}

The main objective of our pulse checking approach is to reliably detect for pulselessness in a patient's carotid arteries. Since the approach is intended to operate at a high level of automation, it must be able to receive Doppler echoes from the carotid arteries (which run relatively parallel to the patient's neck surface) without precise knowledge of the vessel depth. In view of this range uncertainty in the origin of the Doppler echoes, it is suitable to use the continuous-wave (CW) Doppler excitation mode for carotid pulse assessment since this excitation mode has good flexibility in detecting blood flow over a wide depth range. Note that it is also possible to use the pulsed Doppler excitation mode along with long range gates for the assessment. However, this mode was not pursued in our pilot study because

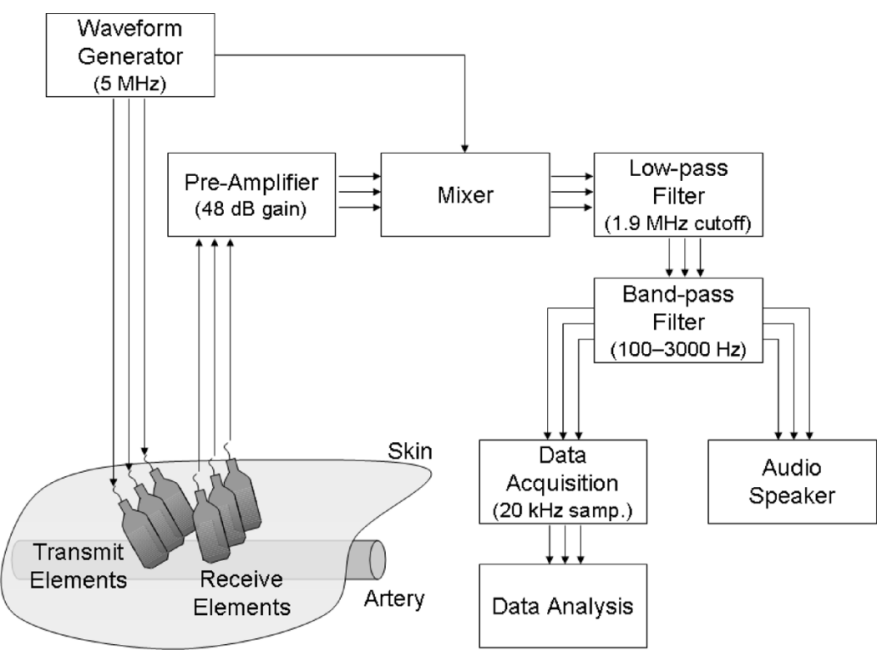

Fig. 1. Schematic diagram of the experimental CW Doppler setup. As a way to improve the flow detection capabilities, three $\mathrm{CW}$ transducer pairs are used so that signals from the artery can be more likely received.

the CW Doppler mode is simpler to implement on hardware and is inherently not abided to maximum depth constraints that arise from the use of range gates.

\section{Overview of Experimental System}

Based on the Doppler ultrasound principles and the design considerations discussed earlier, we developed a CW Doppler experimental setup for carotid pulse assessment. This system is schematically shown in Fig. 1, and it is a modification from the prototype that we previously developed [13]. The major modification seen in the new setup is the use of multi-channel data acquisition as a way to improve the system's flow detection capabilities. In particular, instead of using a single transducer pair at the front end (i.e., a single channel system), the new setup uses an array of three custom-made PZT transducer pairs (5 $\mathrm{MHz}$ center frequency, $-6 \mathrm{~dB}$ bandwidth of $2 \mathrm{MHz}$ ) for $\mathrm{CW}$ Doppler data acquisition. The transmit and receive elements of each transducer pair in the array are respectively angled at $15^{\circ}$ and $30^{\circ}$ with reference to the skin surface so that the beams are not perpendicular to the flow direction in the carotid arteries. During operation, the transducer array can be attached to the patient's neck surface, and it is expected that at least one of these transducer pairs can receive Doppler echoes from the carotid arteries. Note that only three transducer pairs were deployed in our setup to ensure that the transducer array can fit onto the swine necks when conducting our pilot experiments (to be described in later sections), but in general more transducer pairs may be used to increase the chance of locating the patient's carotid arteries.

In terms of the signal acquisition electronics used in the setup, the transmit elements are excited by a $\mathrm{CW}$ waveform generator (Agilent Technologies, Palo Alto, CA, USA; Model 33250A) that is set to a $5 \mathrm{MHz}$ operating frequency, while the echoes picked up at the receive elements are amplified with a cascaded pre-amplifier setup (Minicircuits, Brooklyn, NY, USA; Models ZFL-500LN and ZFL-500) that has an overall gain of $48 \mathrm{~dB}$. Subsequently, to perform demodulation, the pre-amplified ultrasound echoes are passed into a mixer (Minicircuits; Model $\mathrm{ZP}-3 \mathrm{MH}$ ) that receives the carrier signal from the same $\mathrm{CW}$ 
waveform generator used for the transmit elements. It should be noted that quadrature (or complex) demodulation, which can provide directional flow information, is not implemented in this test setup since the flow direction is not essential in detecting the absence or presence of a carotid pulse (in fact, carotid flow is relatively unidirectional). After the ultrasound echoes are mixed with the carrier signal, they are sent into an analog lowpass filter (Minicircuits; Model BLP-1.9) with a $1.9 \mathrm{MHz}$ cutoff frequency to obtain the Doppler signal. Since clutter may be present, the Doppler signal is subsequently forwarded into an analog bandpass filter (Krohn-Hite Corporation, Brockton, MA, USA; Model 3384) that has a passband between $100 \mathrm{~Hz}$ and $2.5 \mathrm{kHz}$. Note that the bandpass filter is used for wall filtering in place of a highpass filter because the Doppler signal quality can be further improved by removing the white noise in the higher Doppler frequency bands (i.e., the bands beyond the range of induced Doppler shifts). In the last hardware stage, the filtered Doppler signal of each transducer channel is digitized at a $20 \mathrm{kHz}$ sampling rate using a data acquisition card (National Instruments, Austin, TX, USA; Model NI6036E) and recorded for aperiodicity analysis. The filtered signal is also fed into a multi-channel audio speaker for real-time qualitative assessment of the Doppler signal quality.

\section{Pulselessness Detection With APERIODICITY ANALYSIS}

\section{A. Spectral Pattern Extraction}

In order to study the level of aperiodicity seen in the Doppler frequency contents, a spectral pattern first needs to be derived from the Doppler signal of each transducer channel. As pointed out by Evans and McDicken [14, Ch. 8], the spectral pattern can typically be obtained by finding time-variant quantities such as the mean and maximum Doppler frequencies. Alternatively, for our carotid pulse assessment approach, we chose to make use of the short-time Doppler power (i.e., the mean-squared Doppler signal value over a sliding time window) as the spectral pattern because this measure does not require direct computation of the Doppler spectrogram and is independent of the frequency biasing effects of noise and wall filter. Also, as a more diversified way of analyzing the Doppler signal, we computed multiple spectral patterns for each transducer channel by finding the short-time Doppler power in different Doppler frequency bands through a filter bank implementation.

\section{B. Analysis Considerations}

In Doppler flow studies, the periodic variation of Doppler frequency contents is well recognized as an evident sign of flow pulsation. Based on this principle, a number of quantitative indices have been derived to evaluate the degree of flow pulsatility from a given spectral pattern (which is often the mean or maximum Doppler frequency over time). For instance, the pulsatility index and the resistance index are two commonly used measures for studying flow pulsatility [14, Ch. 9]. These indices are normalized ratios computed directly from the peaks and troughs of the spectral pattern, and thus their stability inherently depends on whether consistent estimates of the Doppler spectral pattern are available. Also, since these indices are intended for analyzing flow pulsatility, they do not provide direct assessment on the level of pulselessness (i.e., these indices simply consider pulselessness as the reciprocal of pulsatility). In view of such limitations, we considered the development of alternative waveform analysis approaches.

From a signal processing perspective, waveform analysis can be analyzed using both parametric and non-parametric approaches. As pointed out by Stoica and Moses [15, pp. 85], it is generally accepted that parametric methods, such as autoregressive modeling, can more accurately estimate the periodicity in a waveform because of their rigorous signal model assumptions. However, these methods may run into estimation stability problems when they are applied to aperiodic signals because of potential incompatibility of model assumptions. In contrast, non-parametric analysis approaches, such as correlation and Fourier analysis, make few assumptions with regards to the characteristics of the signal, and hence they appear to be more suitable for aperiodicity analysis. Based on this rationale, we recently reported the development of a new pulsation index using Fourier analysis [13]. Nevertheless, we found that this index is not very sensitive at low Doppler signal levels, and it is also not a direct measure of pulselessness. As such, in this paper, we derived a new quantitative index for aperiodicity and applied it to our Doppler-based carotid pulse assessment approach.

\section{The Pulselessness Indicator}

Our new index, which we refer to as the pulselessness indicator, is primarily based on the analysis of aperiodicity seen in different segments of each Doppler spectral pattern (i.e., frequency-banded Doppler power). The analysis makes use of a time-domain signal processing method called the average magnitude difference function (AMDF) that was originally used in speech recognition [16] and is still currently being used by speech researchers [17]. For a segment length of $N$ samples, the normalized AMDF for a pattern segment $x(n)$ is mathematically defined as:

$$
\operatorname{AMDF}(L)=\frac{1}{\mathrm{AMDF}_{\max }}\left[\frac{1}{N-L} \sum_{n=0}^{N-L-1}|x(n)-x(n+L)|\right]
$$

where $L$ is a time lag and $\mathrm{AMDF}_{\text {max }}$ is the maximum AMDF value seen in all lags. As seen in (1), the AMDF describes the difference between a waveform segment and its shifted forms for all possible lags (from 0 to $N$ ), and it can be perceived as a variation of the autocorrelation function that is well-known in signal analysis. The main reason that we chose to compute the AMDF as opposed to the autocorrelation is because the AMDF is a direct measure of waveform dissimilarity [18, pp. 60-63], and thus it is more suitable for studying the level of aperiodicity in a signal. Besides this reason, the AMDF also has the computational advantage of not involving multiplications and complex arithmetic during processing. In terms of its properties, the AMDF has high values at time lags where the time-shifted segment and the original are dissimilar, and it has low values at time lags where the time-shifted segment is similar to the original. Hence, as illustrated in Fig. 2(a) for a periodic pattern segment, 
(a) Periodic Pattern Segment

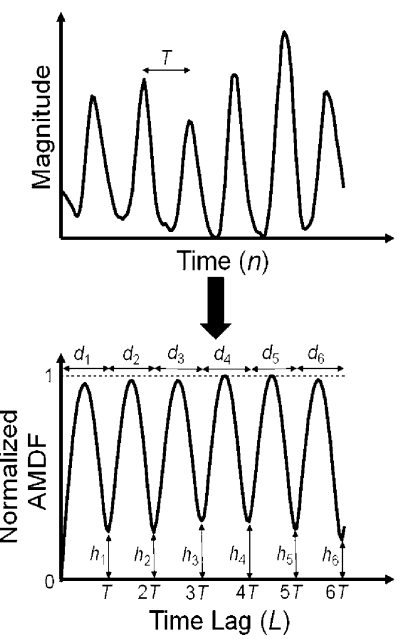

(b) Aperiodic Pattern Segment

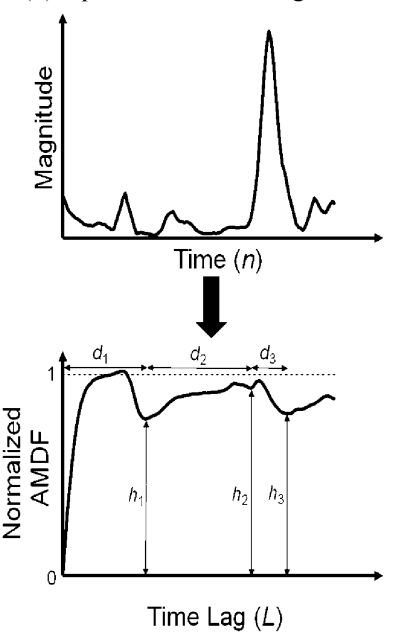

Fig. 2. Characteristics of the AMDF are shown for (a) periodic pattern segments and (b) aperiodic pattern segments (obtained from recordings in vivo). $h_{k}$ and $d_{k}$ respectively denote the trough magnitude and the inter-trough distance. Also, $T$ denotes the pattern period.

the AMDF has troughs that are equally spaced by the spectral pattern period, and the magnitudes of these troughs are small. On the other hand, for an aperiodic pattern segment, few aperiodically spaced troughs are seen in the AMDF, and their magnitudes remain high (as seen in Fig. 2(b).

From the properties of the AMDF, we defined the pulselessness indicator for each pattern segment as a weighted combination between the trough magnitudes and the inter-trough distances seen in an AMDF. Mathematically, it is defined as the following two-term product:

$$
I_{\text {pulselessness }}=w_{\text {mag }} \times w_{\text {dist }}
$$

where $w_{\text {mag }}$ and $w_{\text {dist }}$ are the respective indicator weights of the trough magnitudes and the inter-trough distances. For the $w_{\text {mag }}$ weight, it is defined as the mean magnitude of all troughs in the normalized AMDF; as for the $w_{\text {dist }}$ weight, it is set equal to the fraction of AMDF troughs that are not periodically spaced with their adjacent troughs. Given that the AMDF has $M$ troughs, these weight definitions can be mathematically expressed as follows:

$$
\begin{aligned}
w_{\text {mag }} & =\frac{\sum_{k=1}^{M} h_{k}}{M} \\
w_{\text {dist }} & =\frac{\operatorname{dim}\left\{k: d_{k} \neq d_{k+1} \mid k \in[1, M]\right\}}{M}
\end{aligned}
$$

where $h_{k}$ and $d_{k}$ are the trough magnitudes and inter-trough distances as shown in Fig. 2, and the $\operatorname{dim}\{$.$\} notation refers to the$ number of elements in the set. From (3a) and (3b), it can be seen that if a pattern segment is aperiodic, then the pulselessness indicator approaches unity because $w_{\text {mag }}$ and $w_{\text {dist }}$ both have values close to unity for this case. Conversely, if the pattern segment is periodic, then the pulselessness indicator approaches zero. It is worth noting that the above definition for pulselessness indicator might give rise to false-positive estimates in cases where the carotid pulse is only quasi-periodic. Nevertheless, by using

this strict definition, the risk of having false-negative pulselessness diagnoses would be lower (which is an important attribute in medical emergencies since rescuing life is of prime concern).

Since multiple transducer channels are used and a number of Doppler spectral patterns are extracted from each channel, many pulselessness indicator values can be obtained for each analysis time segment. Thus, a selection rule is needed to identify the indicator value that most accurately reflects the carotid pulse state. It is preferable to choose the minimum of all indicator values because this selection rule can ensure that pulselessness is detected in all the channels, thereby reducing the number of false-positive pulselessness diagnoses. Based on this principle, we defined the overall pulselessness indicator as the smallest indicator value amongst the Doppler spectral patterns from all transducer channels, and this quantity is simply referred to as the pulselessness indicator from hereon to maintain simplicity of discussion.

\section{In Vivo Performance Assessment Method}

\section{A. Experimental Protocol}

To assess the performance of the Doppler-based carotid pulse assessment approach, we conducted a series of cardiac arrest experiments in vivo with five domestic swines that weighed about $30 \mathrm{~kg}$ each. In each experiment, the swine was first anestheized and intubated according to the approved animal study guidelines. Subsequently, cardiac arrest was externally induced on the swine by applying a low-voltage electrical shock at the surface of the swine heart to trigger ventricular fibrillation. After at least $15 \mathrm{~s}$ (and at most $2 \mathrm{~min}$ ) from the onset of cardiac arrest, a defibrillating shock at a strength of $120 \mathrm{~J}$ was applied to the swine heart, and subsequent shocks at strengths of $150 \mathrm{~J}$ and $200 \mathrm{~J}$ were applied as necessary if blood circulation was not resumed in the swine. Throughout this entire procedure, the Doppler signal was acquired at the swine's carotid arteries using our multi-channel CW Doppler setup, and the ECG was simultaneously recorded. As the ground truth of the carotid pulse state during the procedure, the aortic pressure was also measured invasively by inserting an intravascular catheter into the swine's aorta. The same experimental procedure was then repeated several times on each swine, but a 3 min time interval was placed in between each procedure to allow for re-stabilization of the swine's cardiac activity. This in vivo study protocol was conducted under the approval of the animal ethics committee at the University of Colorado.

As a comparative assessment of the multi-channel Dopplerbased pulse checking approach, we obtained Doppler data in vivo from another set of cardiac arrest experiments conducted on five other swines. These series of Doppler data were acquired using the single-channel prototype that we previously reported [13]. The procedure for these experiments, which were performed with approval from the animal ethics committee at the University of Iowa, was similar to the one used in the multichannel carotid pulse assessment studies. However, instead of measuring the aortic pressure, this second series of experiments measured the carotid arterial pressure as the ground truth of the pulse state. Also, in the single-channel experiments, a sternotomy operation was performed on the swine to expose its 


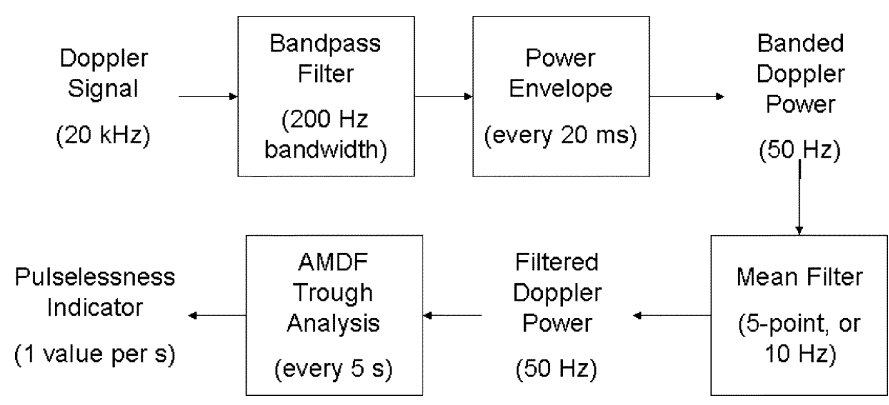

Fig. 3. Flow diagram of the Doppler data analysis scheme. During the analysis, this scheme is repeated for all the bandpass filters in the filter bank.

heart and a defibrillating shock was directly applied to the heart using hand-held surgical electrode paddles.

\section{B. Data Analysis Procedure}

From the acquired Doppler data, the pulselessness indicator of different analysis time segments was computed using Matlab (Mathworks Inc., Natick, MA, USA; Version 7.0). As illustrated in Fig. 3, the computation process first involves the use of a digital filter bank to extract the spectral pattern (i.e., the shorttime Doppler power) in different Doppler frequency bands of each transducer channel. In our analysis, each filter in the filter bank was implemented as a finite impulse response band-pass filter (128th-order, Hamming window design method), and the short-time Doppler power over every $20 \mathrm{~ms}$ was found in five nominal Doppler frequency bands: $400-600 \mathrm{~Hz}, 800-1000 \mathrm{~Hz}$, 1200-1400 Hz, 1600-1800 Hz, 2000-2200 Hz. Note that these frequency bands were chosen for the analysis since the Doppler signal acquired by the experimental setup has a passband between 100-2500 Hz. After the Doppler spectral patterns were calculated, they were passed through a 5-point moving average filter to remove random noises in the spectral patterns. Subsequently, AMDF trough analysis was performed on the filtered Doppler spectral patterns over a $5 \mathrm{~s}$ diagnostic time window in order to compute the pulselessness indicator value, and this analysis was repeated at $1 \mathrm{~s}$ time window increments. During the AMDF analysis, the trough locations were determined by a first derivative test that defines a trough as an AMDF data point where its seven preceding and succeeding AMDF samples have decreasing and increasing slopes respectively. Also, in computing the aperiodicity between inter-trough distances, the troughs with normalized magnitudes greater than 0.5 were not included in order to avoid the spurious noise troughs.

\section{Performance Assessment Results}

\section{A. Pulse State Traces}

Fig. 4 shows the pulselessness indicator trace computed from the multi-channel Doppler data of an experiment where the swine suffered a $15 \mathrm{~s}$ cardiac arrest. For comparison purposes, this figure also shows the ECG trace, the aortic pressure, and the Doppler spectrograms of all transducer channels. It can be seen from these plots that the pulselessness indicator values (which were computed over a 5 s sliding time window) are high during the cardiac arrest and are low before the cardiac arrest as well as after the defibrillation shock. This result is consistent with the aortic pressure trace that shows a significant drop during the cardiac arrest. Another observation to be noted from Fig. 4 is that the pulselessness indicator's specificity (i.e., ability to distinguish normal states from cardiac arrest states) was not affected even when transducer $\#_{2}$ did not consistently receive flow echoes from the swine's carotid arteries. This finding is a result of the fact that we defined the pulselessness indicator as the minimum indicator value seen in all the transducer channels.

As a comparison to the multi-channel results, Fig. 5 shows the pulselessness indicator trace calculated from a set of singlechannel Doppler data where the transducer pair was not well placed near the carotid arteries during the cardiac arrest experiment. In this case, the pulselessness indicator still shows high values during the cardiac arrest (also between 15-30 s), but it is not always low during normal states. This finding suggests that the pulselessness indicator's performance is more prone to the transducer off-positioning problems when only a single transducer pair is used. Consequently, the indicator may have a higher false alarm rate when it is applied to single-channel Doppler data.

\section{B. Receiver Operating Characteristics}

To study the sensitivity and specificity of the pulselessness indicator, we classified the indicator estimates obtained from all the experiments into two categories: one corresponding to cardiac arrest state and the other to normal state. The classification decision was based on the ground truth that we obtained from the aortic pressure (for the multi-channel data) and the arterial pressure (for the single channel data). In particular, the indicator estimates were classified into the cardiac arrest category if the aortic/arterial pressure over the $5 \mathrm{~s}$ diagnostic time was low and non-pulsatile, and they were classified into the normal category if pulsatile trends are seen in the pressure. Note that, for the experiments with the multi-channel setup, a total of 2807 indicator estimates were classified into the normal category, while 1030 estimates were classified into the cardiac arrest category; as for the experiments with the single-channel setup, 5667 indicator estimates were classified as normal and 1619 estimates were classified as cardiac arrest. From the classified estimates, histograms were constructed to analyze the distribution of indicator values corresponding to normal and cardiac arrest states. These histograms were subsequently used to evaluate the indicator's receiver operating characteristics (i.e., pulselessness detection probability as a function of false alarm rate).

Fig. 6(a) and (b) respectively show the histograms compiled from all the pulselessness indicator estimates obtained with the multi-channel and single-channel experimental setups, and their corresponding distribution statistics are summarized in Table I. As shown in these figures, the pulselessness indicator values for the multi-channel setup has a minor degree of overlap between cardiac arrest and normal states, while for the single-channel setup, the overlap between the indicator values of the two categories is larger. To further support and quantify this finding, the receiver operating characteristic curves of the multi-channel and single-channel setups are plotted in Fig. 7. From these curves, it can be seen that the multi-channel setup has a slightly better sensitivity and specificity than the single-channel setup. For instance, at a given false alarm rate of 0.05 (or $95 \%$ specificity), 


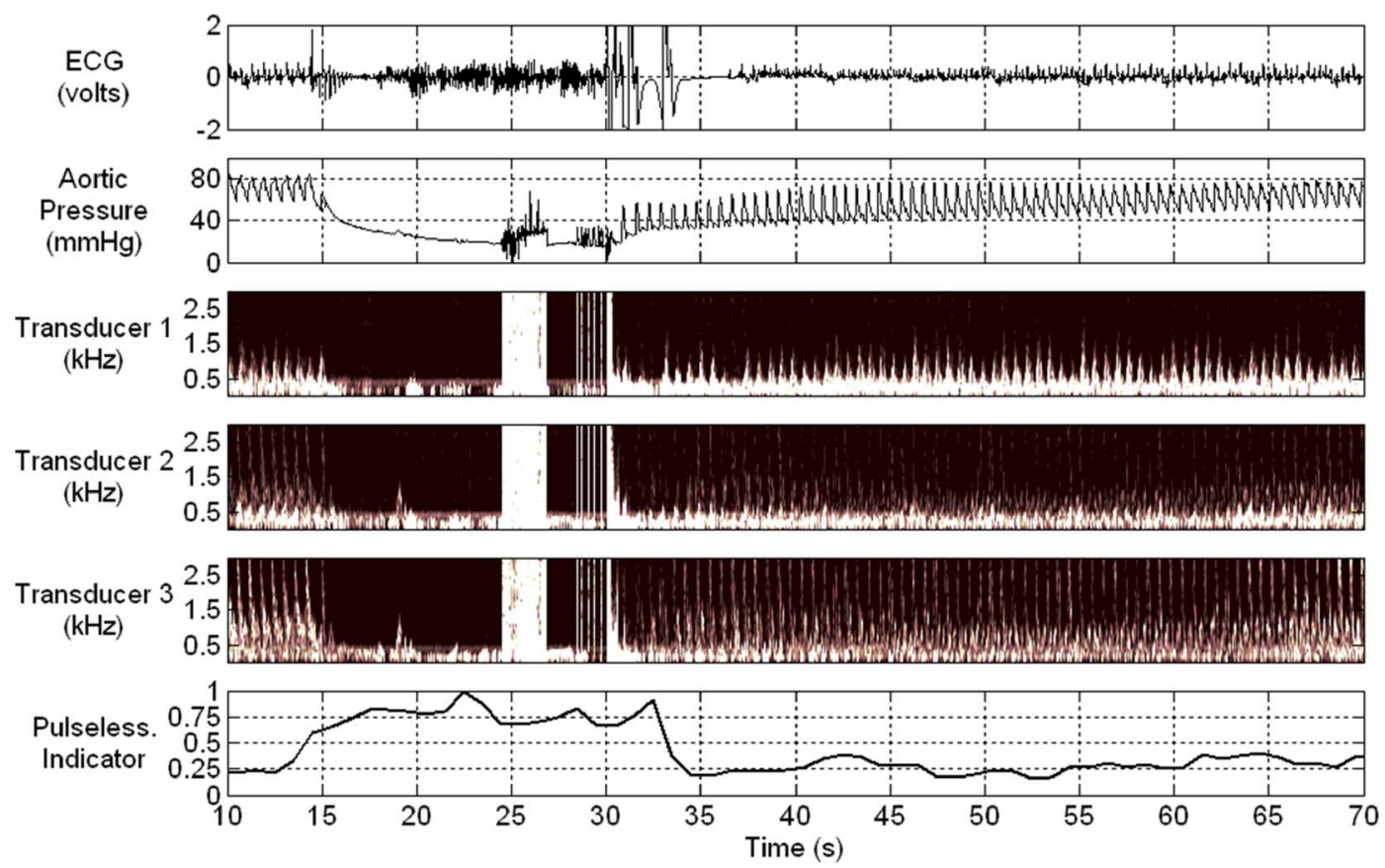

Fig. 4. Pulselessness indicator values computed from a set of multi-channel Doppler data acquired during a swine cardiac arrest experiment. As a reference, the ECG, aortic pressure, and Doppler spectrograms of each transducer channel are also provided. In this experiment, the swine was in a cardiac arrest between 15-30 s. Note that the jamming noise seen in the Doppler spectrograms at about $25 \mathrm{~s}$ (just prior to the shock) is due to the pre-charging of the defibrillator.

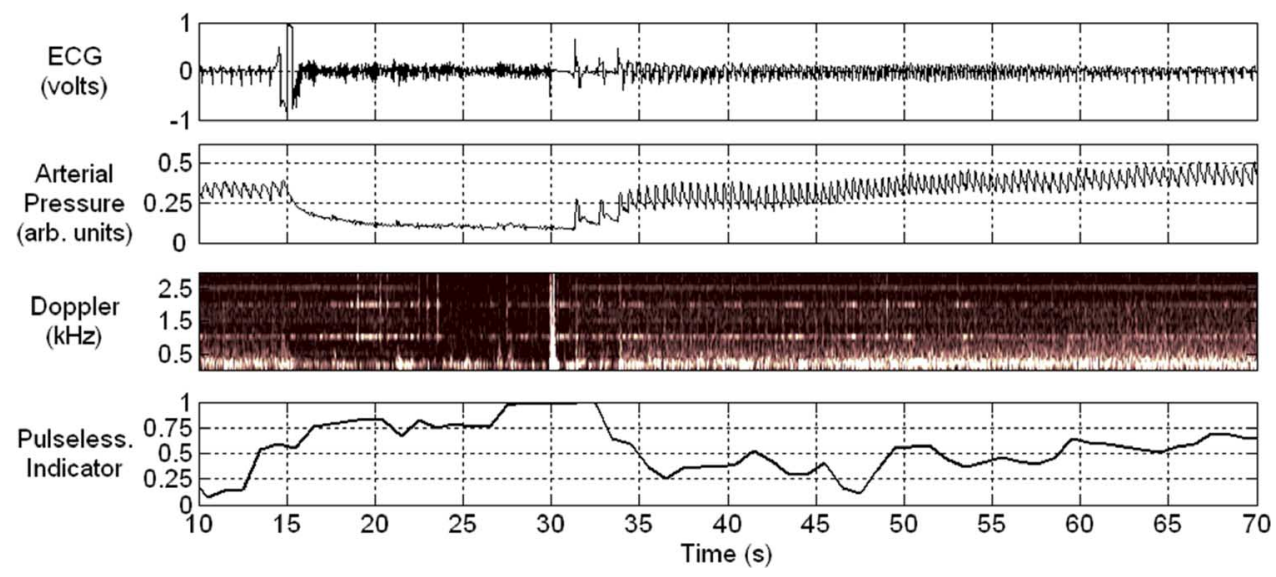

Fig. 5. The ECG, arterial pressure, Doppler spectrogram, and pulselessness indicator values corresponding to a cardiac arrest experiment where the single-channel transducer pair was partially off-positioned from the swine's carotid arteries. In this experiment, cardiac arrest was induced on the swine between 15-30 s.

the multi-channel setup has a detection probability of around 0.98 (or $98 \%$ sensitivity), while the single-channel setup has a detection probability of about 0.92 (or $92 \%$ sensitivity). Nevertheless, regardless of whether the pulselessness indicator is derived from the single-channel or multi-channel setup, this new measure has demonstrated better sensitivity than the manual pulse check which only achieved sensitivities of $18 \%$ and $58 \%$ respectively by lay rescuers [6] and paramedics [7].

\section{Other CASE Study Results}

\section{A. Monitoring in Presence of Pulseless Electrical Activity}

As a further analysis, we performed additional swine cardiac arrest experiments in vivo that corresponded to clinically relevant scenarios and applied the Doppler-based carotid pulse checking approach to monitor the resuscitation progress. The experimental procedure used for these additional case studies was similar to the one described in Section IV. The only procedural difference between these additional experiments and the ones described earlier is that the defibrillation shocks were not applied to the swine until at least 5 min after the cardiac arrest onset in order to more realistically simulate the emergency response time of lay rescuers.

We first considered a case where PEA was seen after defibrillation. In this study, fibrillation was induced on the swine after $60 \mathrm{~s}$ from the start of experiment, and a $120 \mathrm{~J}$ defibrillation shock was delivered at 490 s. Fig. 8 shows the corresponding ECG, aortic pressure, Doppler spectrograms, and pulselessness indicator traces of this study for the period between $480-540 \mathrm{~s}$. As 
(a) Multi-Channel Setup

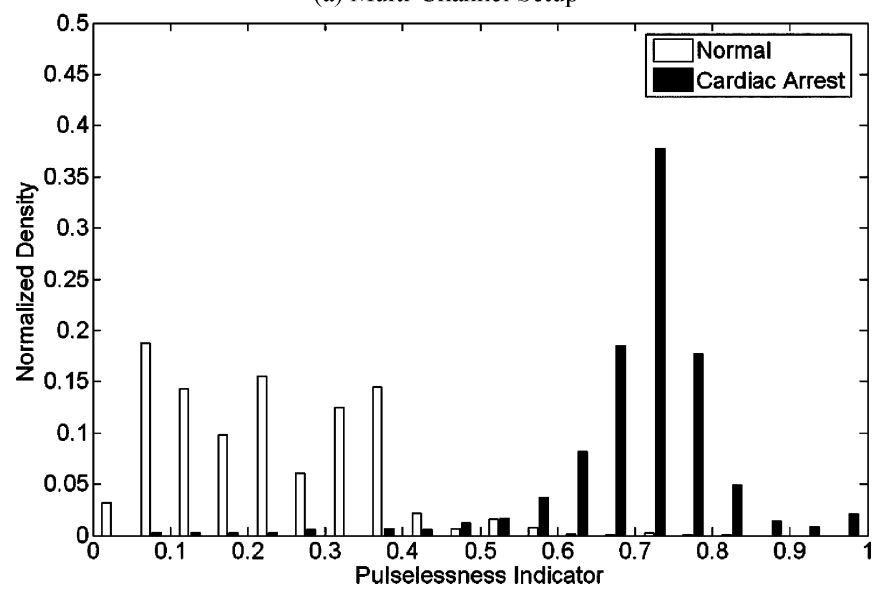

(b) Single-Channel Setup

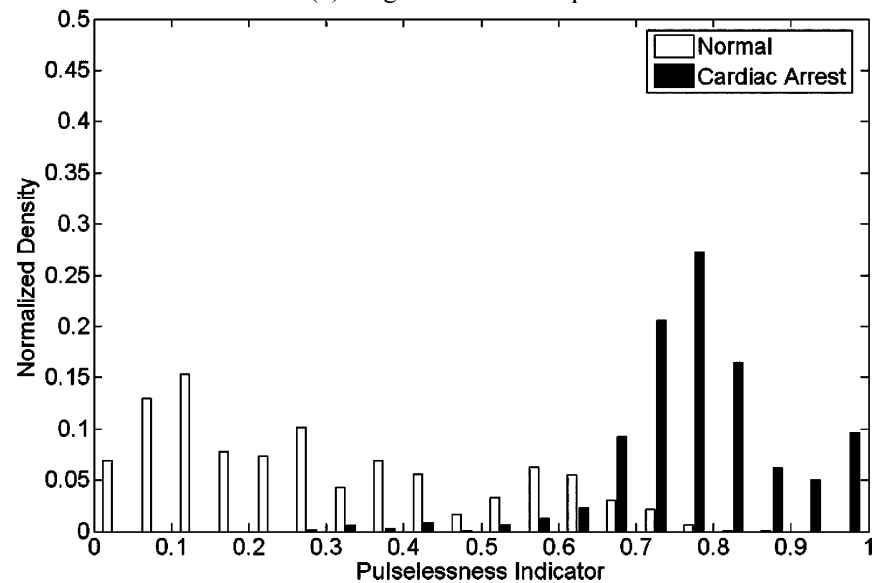

Fig. 6. Distribution of the pulselessness indicator estimates for: (a) multichannel setup and (b) single-channel setup. The white and black histogram bars respectively correspond to the normal and cardiac arrest states.

TABLE I

PULSELESSNESS INDICATOR ESTIMATES FOR MULTI- AND SINGLE-CHANNEL DOPPLER DATA

\begin{tabular}{c|c|c|c|c}
\hline & $\begin{array}{c}\text { Physiological } \\
\text { State }\end{array}$ & $\begin{array}{c}\text { Total Number of } \\
\text { Estimates }\end{array}$ & $\begin{array}{c}\text { Mean Indicator } \\
\text { Value }\end{array}$ & $\begin{array}{c}\text { Standard } \\
\text { Deviation }\end{array}$ \\
\hline \multirow{3}{*}{ Multi-Channel } & Normal & 2807 & 0.223 & 0.129 \\
\cline { 2 - 5 } & Cardiac Arrest & 1030 & 0.708 & 0.104 \\
\hline \multirow{2}{*}{ Single-Channel } & Normal & 5667 & 0.289 & 0.206 \\
\cline { 2 - 5 } & Cardiac Arrest & 1619 & 0.782 & 0.108 \\
\hline
\end{tabular}

can be seen, the defibrillation shock successfully restored regular electrical activity in the swine, but the pulselessness indicator remained at a high value after the shock. These observations suggest that the swine was experiencing post-defibrillation pulseless electrical activity and needed further CPR. Note that this diagnostic decision is consistent with the unchanged aortic pressure (i.e., our ground truth) seen during this period.

\section{B. Monitoring for Recovery After CPR}

In another case, we examined the resuscitation progress during and after a CPR procedure. The swine involved in this study was fibrillated after $40 \mathrm{~s}$ from the start time, and

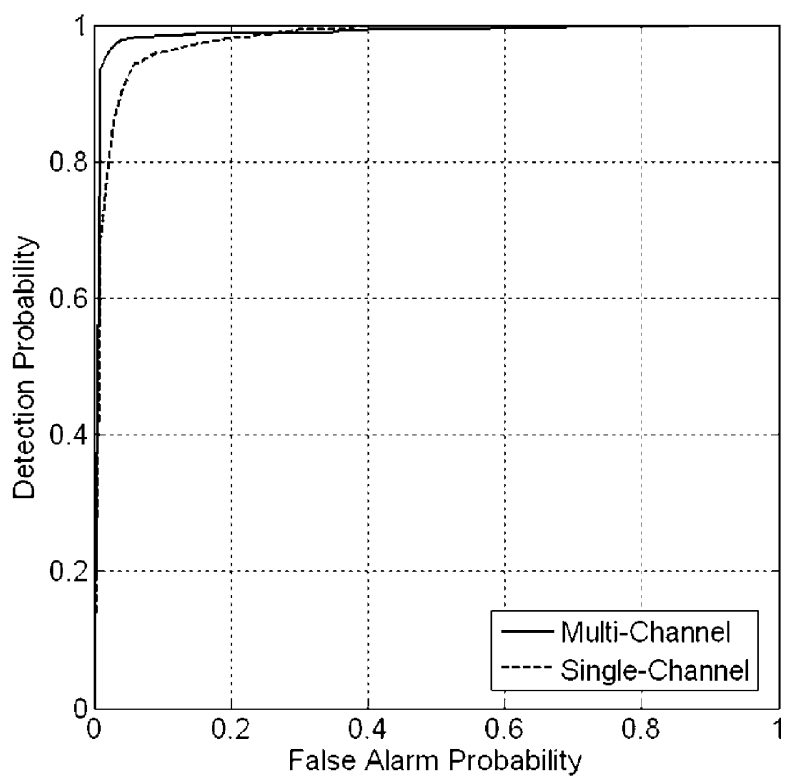

Fig. 7. Receiver operating characteristics of the multi- channel and singlechannel Doppler carotid pulse checking approaches.

at $340 \mathrm{~s}$, it was defibrillated with a $120 \mathrm{~J}$ electrical shock. After the shock delivery, PEA was observed in the swine, and thus CPR was performed on the swine starting at $420 \mathrm{~s}$. The CPR procedure was initially done for $30 \mathrm{~s}$, but after seeing no signs of recovery in the aortic pressure, the procedure was repeated for three times during the following time intervals: $460-510 \mathrm{~s}, 550-610 \mathrm{~s}$, and $670-700 \mathrm{~s}$. After the last set of chest compressions, the swine's carotid pulse gradually returned. The corresponding physiological traces during and after the last CPR procedure are respectively shown in Fig. 9 (between 650-710 s) and Fig. 10 (between 710-770 s). As indicated by the pulselessness indicator trace in Fig. 10, the gradual return of the swine's carotid pulse after $715 \mathrm{~s}$ was detected by our method. This observation shows that it is possible to use our method to monitor the resuscitation progress after CPR procedures. On another note, the pulsatile flow seen during a CPR procedure is not consistently detected by our method as seen in Fig. 9. This result is likely attributed to the fact that the spectral Doppler echoes received by the transducers were only quasi-periodic in time (as shown in the Doppler spectrograms), and hence the pulselessness indicator did not show particularly low values during the CPR between $670-700$ s. To account for these quasi-periodic artifacts, it is necessary to use a more generalized definition of pulselessness for our data analysis (though such a solution is not considered in this pilot study).

\section{CONCLUSION}

This pilot study has been set out to evaluate the use of Doppler ultrasound for carotid pulse assessment and examine the performance of a new pulselessness indicator that we developed. In our study, the Doppler-based carotid pulse checking approach, together with the pulselessness indicator, has demonstrated consistent performance for pulse state assessment. In particular, the in vivo results presented in this paper have indicated that our approach has both high sensitivity and specificity for short diagnostic time windows of $5 \mathrm{~s}$. Such a finding suggests that the 


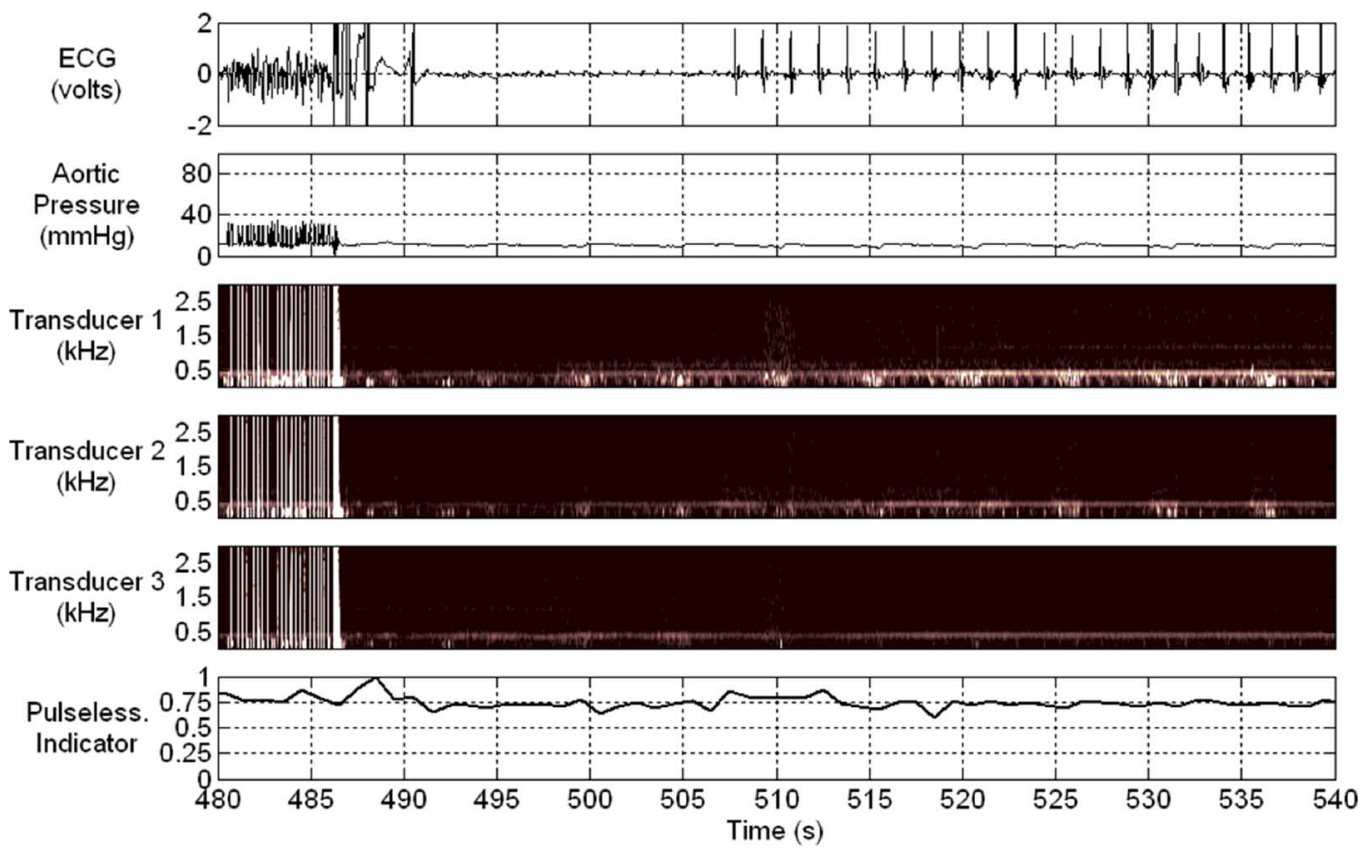

Fig. 8. The pulselessness indicator trace for a case where post-shock pulseless electrical activity is observed in the swine (after $505 \mathrm{~s}$ ). For comparison purposes, the ECG, aortic pressure, and Doppler spectrograms are also plotted.

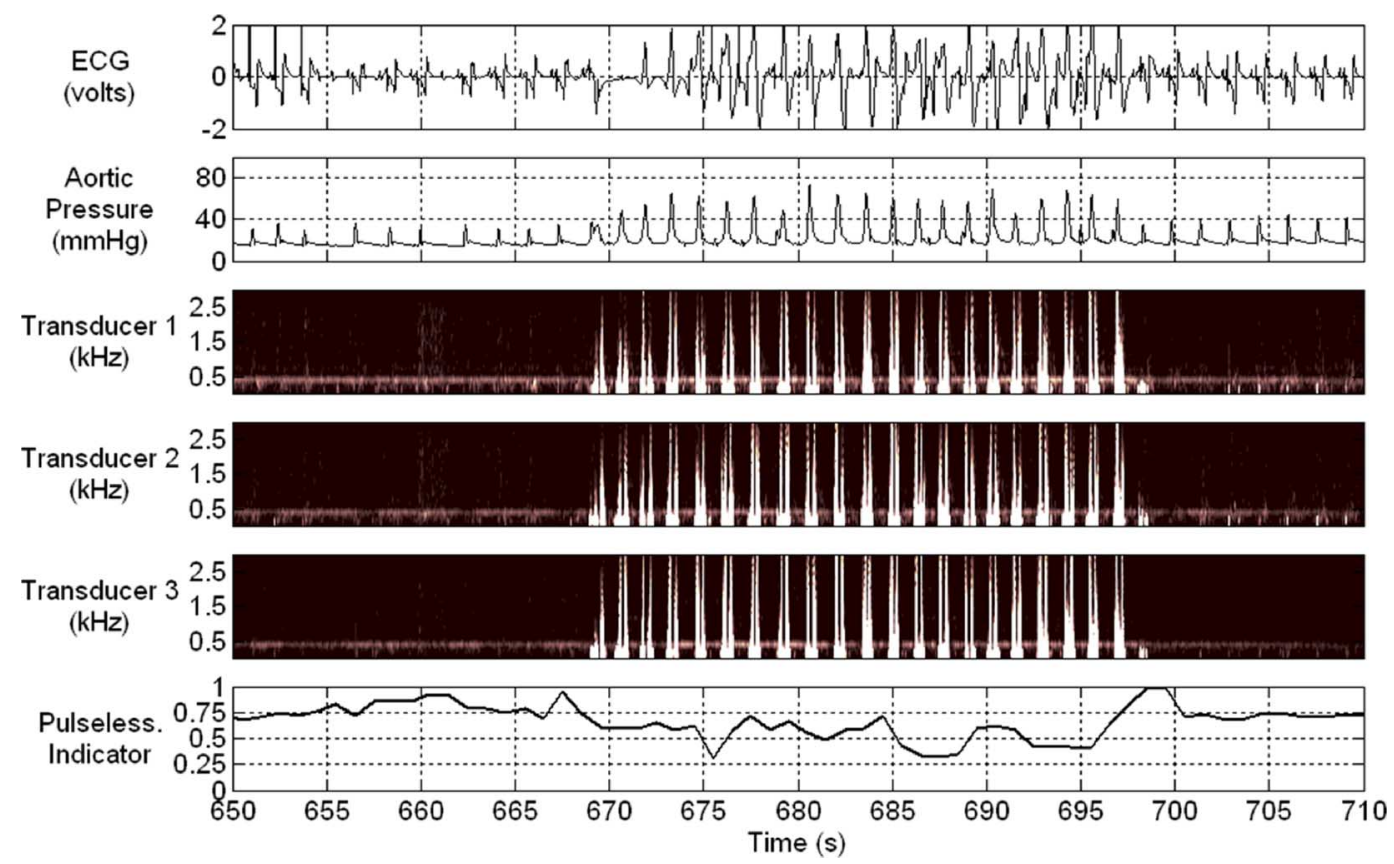

Fig. 9. The pulselessness indicator trace for a case where a CPR procedure was performed to the swine (between $670-700 \mathrm{~s}$ ).

Doppler-based pulse checking method can potentially be used in place of the manual pulse check to monitor the resuscitation progress and in turn improve the survival chances of cardiac arrest patients.

One potential limitation of our study relates to the fact that we defined the pulselessness indicator as the level of aperiodicity observed in the spectral Doppler contents. Such a definition allows the indicator to have high sensitivity for pulselessness, but it might affect the indicator's specificity under circumstances where the carotid pulse is only quasi-periodic in nature. For in- stance, as illustrated in Fig. 9, the pulselessness indicator values are not particularly low for the quasi-periodic pulse state seen during CPR. If this quasi-periodic pulse is to be considered as a sign of regular cardiac activity, then our aperiodicity definition for the pulselessness indicator may need to be modified.

Parts of our next steps in the development process will be to design an advanced version of our transducer array to support hands-free operation after its initial attachment to the patient's neck surface. In particular, it is envisioned that the transducer pairs can be designed as a flat sensor pad that can be placed 


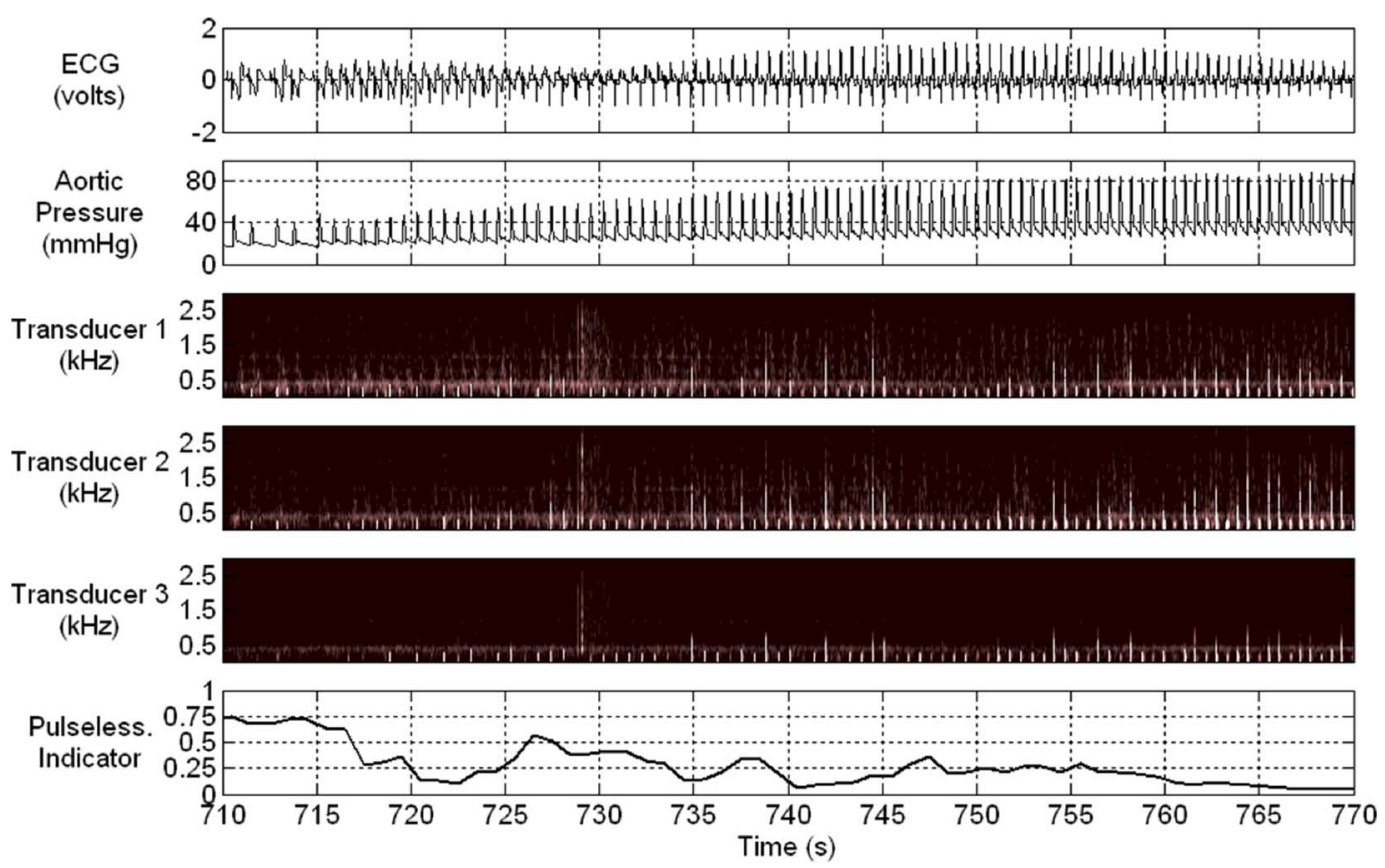

Fig. 10. The pulselessness indicator trace for a case where the swine's carotid pulse gradually returned after a series of CPR procedures. This trace follows from the one shown in Fig. 9.

on the skin surface like a bandage. Such a transducer design should improve our system's portability and ease-of-use in an emergency setting.

\section{REFERENCES}

[1] Heart Disease and Stroke Statistics-2006 Update. Dallas, TX: American Heart Assoc., 2006.

[2] J. Engdahl, A. Bang, J. Lindqvist, and J. Herlitz, "Factors affecting short- and long-term prognosis among 1069 patients with out-of-hospital cardiac arrest and pulseless electrical activity," Resuscitation, vol. 51, pp. 17-25, 2001.

[3] D. C. Parish, K. M. D. Chandra, and F. C. Dane, "Success changes the problem: Why ventricular fibrillation is declining, why pulseless electrical activity is emerging, and what to do about it," Resuscitation, vol. 58, pp. 31-35, 2003.

[4] "International resuscitation guidelines, "Part 6: Advanced cardiovascular life support. Section 7: Algorithm approach to ACLS emergencies"," Resuscitation, vol. 46, pp. 163-193, 2000.

[5] B. Eberle, W. F. Dick, T. Schneider, G. Wisser, S. Doetsch, and I. Tzanova, "Checking the carotid pulse check: Diagnostic accuracy of first responders in patients with and without a pulse," Resuscitation, vol. 33, pp. 107-116, 1996.

[6] P. Moule, "Checking the carotid pulse: Diagnostic accuracy in students of the healthcare professions," Resuscitation, vol. 44, pp. 195-201, 2000.

[7] F. Lapostolle, P. Le Toumelin, J. M. Agostinucci, J. Catineau, and F. Adnet, "Basic cardiac life support providers checking the carotid pulse: Performance, degree of conviction, and influencing factors," Acad. Emerg. Med., vol. 11, pp. 878-880, 2004.

[8] M. M. Hayes, R. A. Berg, and C. W. Otto, "Monitoring during cardiac arrest: Are we there yet?," Curr. Opin. Crit. Care, vol. 9, pp. 211-217, 2003.

[9] C. W. Hallaway and J. J. Menegazzi, "Waveform analysis of ventricular fibrillation to predict defibrillation," Curr. Opin. Crit. Care, vol. 11, pp. 192-199, 2005.

[10] J. T. Niemann, S. J. Stratton, B. Cruz, and R, J. Lewis, "Outcome of out-of-hospital postcountershock asystole and pulseless electrical activity versus primary asystole and pulseless electrical activity," Crit. Care Med., vol. 29, pp. 2366-2370, 2001.

[11] Y. Morimoto, O. Kemmotsu, Y. Morimoto, and S. Gando, "End-tidal carbon dioxide and resuscitation," Curr. Opin. Anaesthesiol., vol. 12, pp. $173-177,1999$.
[12] T. Pellis, J. Bisera, W. Tang, and M. H. Well, "Expanding automatic external defibrillators to include automated detection of cardiac, respiratory, and cardiorespiratory arrest," Crit. Care Med., vol. 30, pp. S176-S178, 2002.

[13] B.I. Raju, E. Cohen-Solal, and S. Ayati, "A novel ultrasound based automated pulsatile flow detection method for resuscitation," in Proc. IASTED Int. Conf. Biomed. Eng., 2005, pp. 325-330.

[14] D. H. Evans and W. N. McDicken, Doppler Ultrasound: Physics, Instrumentation and Signal Processing, 2nd ed. West Sussex, U.K.: Wiley, 2000.

[15] P. Stoica and R. L. Moses, Introduction to Spectral Analysis. Englewood Cliffs, NJ: Prentice-Hall, 1997.

[16] M. Y. Ross, H. L. Shaffer, A. Cohen, R. Freudberg, and H. J. Manley, "Average magnitude difference function pitch extractor," IEEE Trans. Acoust., Speech, Signal Process., vol. 22, pp. 353-362, 1974.

[17] O. Deshmukh, C. Y. Espy-Wilson, A. Saloman, and J. Singh, "Use of temporal information: Detection of periodicity, aperiodicity, and pitch in speech," IEEE Trans. Speech Audio Process., vol. 13, pp. 776-786, 2005.

[18] A. M. Kondoz, Digital Speech: Coding for Low Bit Rate Communication Systems. West Sussex, U.K.: Wiley, 1994.

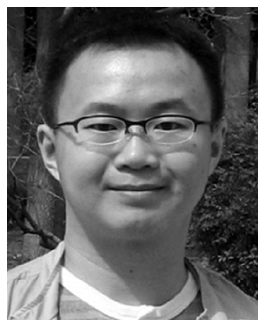

Alfred C. H. Yu (S'99-M'07) was born in Hong Kong in 1980. He received the B.Sc. degree in electrical engineering from the University of Calgary, $\mathrm{AB}$, Canada, in 2002. He then completed the M.A.Sc. and Ph.D. degrees in electrical engineering (biomedical stream) from the University of Toronto, ON, Canada, in 2003 and 2006, respectively. In the summer of 2005, he was a research intern at Philips Research, Briarcliff Manor, NY. For his graduate work, he studied the theoretical aspects of eigen-based signal processing strategies for Doppler ultrasound and color flow imaging. He also worked on the modeling of Doppler ultrasound signals and Doppler spectral broadening. His graduate work was funded by the Canada Graduate Scholarship program from the Natural Sciences and Engineering Research Council of Canada.

He is currently a Postdoctoral Research Fellow and Honorary Assistant Professor in the Department of Electrical and Electronic Engineering, University of Hong Kong. His research interests are broadly centered around the design aspects of medical ultrasound systems. He is also interested in studying the fundamentals and physics of ultrasound. 


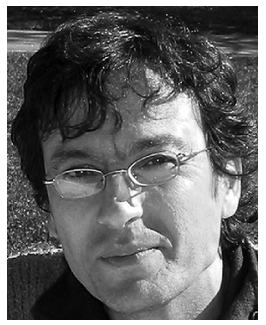

Eric Cohen-Solal received the M.S. and Ph.D. degrees, both in computer science, from University Louis Pasteur, Strasbourg, France, in 1991 and 1996, respectively.

He joined Philips Research North America in 1995 and has worked, since then, in diverse areas including medical image analysis, computer vision for man-machine interaction applications, and more recently, ultrasound-based devices for medical use. His focus is currently on medical informatics.

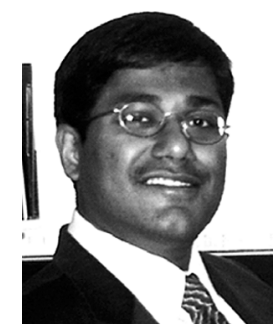

Balasundar I. Raju received the Bachelor's degree in mechanical engineering from the Indian Institute of Technology, Madras, in 1994. He received two Master's degrees in 1998, one in mechanical engineering and the other in electrical engineering and computer science, and a Ph.D. degree in electrical engineering and computer science in 2002, all from the Massachusetts Institute of Technology, Cambridge, MA.

Since 2003, he has been a Senior Member of Research Staff at Philips Research North America, Briarcliff Manor, NY. His research interests include signal and image processing, ultrasound mediated therapy, and tissue characterization.

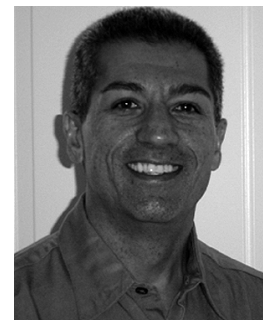

Shervin Ayati received the B.S.E.E. degree in computer engineering in 1985 and the M.S.E.E. degree with concentration in signal processing and communications from Northeastern University, in 1995.

$\mathrm{He}$ is responsible for Strategic Research and Business Development in the Cardiac Care business unit, a division of Philips Medical Systems that is focused on emergency resuscitation, diagnostic cardiology and medical consumables. He was developing industrial and consumer products from 1985 to 1991 . Since 1991, he has been working in R\&D and business development in various medical device companies in cardiology and anesthesiology fields. He has developed patented biphasic waveform for transthoracic defibrillators. He joined Philips Medical Systems in 2001 leading the Advanced Life Support Research. Some of his research was concentrated in detection of brain perfusion in cardiac arrest patients. He holds many patents and has published in various technical and medical journals. 